Associate Professor Esmaeil MEHDIZADEH, PhD

E-mail: emehdi@qiau.ac.ir (Corresponding author)

Department of Industrial Engineering, Faculty of Industrial \&

Mechanical Engineering, Qazvin Branch, Islamic Azad University, Qazvin, Iran

Lecturer Hbibreza GHOLAMI, PhD Student

E-mail:hr_ghlamy@yahoo.com

Department of Industrial Engineering, Faculty of Industrial \&

Mechanical Engineering, Qazvin Branch, Islamic Azad University,

Qazvin, Iran

Associate Professor Bahman NADERI, PhD

E-mail: bahman.naderi@aut.ac.ir

Department of Industrial Engineering, Faculty of Engineering,

Kharazmi University, Tehran, Iran

\title{
A ROBUST OPTIMIZATION MODEL FOR MULTI-PRODUCT PRODUCTION PLANNING IN TERMS OF UNCERTAINTY OF DEMAND AND DELIVERY TIME
}

\begin{abstract}
Production planning in the uncertainty conditions is one of the inherent characteristics of manufacturing environments. In this paper, a new multi-product multi-stage production planning model is presented. The demand and delivery time are considered to be uncertain. In order to deal with non-deterministic parameters and reduce their impact on optimal solution, a robust optimization model with a description of the scenario of uncertainty data has been used. The objective is to minimize the cost over the planning horizon. To solve the proposed model, The Branch and Bound (B\&B) method is used by using Lingo software.
\end{abstract}

Keywords: Production planning, robust optimization, uncertain demand, delivery time.

JEL Classification: I11, C61

\section{Introduction}

Determining the optimal product is one of the main objectives of the production planning in organizations to increase profits and reduce production costs in a longterm horizon, taking into account existing constraints. In fact, the purpose of the

DOI: $10.24818 / 18423264 / 52.4 .18 .15$ 
Esmaeil Mehdizadeh, Habibreza Gholami, Bahman Naderi

problem of determining the optimal product combination is to find a production plan in such a way that, while meeting the existing operational constraints (resource access, demand), the objective function of each organization (cost reduction, increase in profit) is also optimized. But one of the problems in this area is the existence of real-world uncertainties. This means that in such a situation, it is always possible to confront uncertainty, turbulent, incomplete or even mistaken data. In the production planning problem, there are also uncertainties such as the amount of demand, the availability of resources, the delivery time and so on, that it makes the decision to determine the optimal production of each product with challenges. In this research, a robust optimization approach has been used as an approach to uncertainty modeling.

In the research on production area, uncertainty production planning is considered to be essential. After reviewing several articles, Leung et al. stated that many of the real-world production planning problems include inaccurate information. In literature related to this subject, various approaches have been used to deal with various types of ambiguities. Mathematical planning and probabilistic approaches have been used to formulate ambiguities and inaccuracies in manufacturing systems. They provided a possible planning method to solve the multi-site integration planning problem. [2]. Leung and $\mathrm{Wu}$ [4] studied the integration planning problems of which some of the parameters were inaccurate. They provided an optimization model for minimizing production costs in manufacturing systems, and they analyzed their proposed model with one-stage and multi-stage data and examined the transaction between the model's solvability and stability. In 2011, Rozenblit et al. [ ] used a steady interval optimization model to modify the multi-product multi-cast production planning model, which in their model was just the demand factor as an uncertain parameter. They used the simulation method to solve their model. Kastsian and Monnigmann [5] presented an effective method for determining optimal production conditions for automated production systems under uncertainty conditions. They optimized a nonlinear dynamical system with respect to the non-linear objective function using a robust optimization. Sakhaii et al. [6] presented a robust optimization model for linear mix integer programming to solve a dynamic cellular production problem under uncertainty conditions. The model provided by them reduces the cost of machine failure, re-allocation of equipment, training and recruitment of personnel. 
A Robust Optimization Model for Multi-Product Production Planning in Terms of Uncertainty of Demand and Delivery Time

\section{Problem formulation}

In the study of bi-stage production systems, the primary parts (the products of the first part) are manufactured by the first machinery and the products produced in the first part and the parts purchased from outside are assembled in the final product by the second group of machines. In this stage, a mathematical programming model for two-stage production planning based on the model presented by Ramezanian et al. [3] is presented. Then a robust optimization model is proposed for the above-mentioned problems in which some parameters are inaccurate. To create a real model, some parameters such as product demand and delivery time are considered uncertain. The initial model relates to a multi-stage multi-product multi-machinery by-stageal manufacturing system, and it is assumed that the parameters of this model are definite. Decisions on commissioning and pre-order time are considered for the problem to reflect the real environment of the industry. If a particular product is produced at a given time, then each machine associated with the production of that product is set up only once at that time.

\subsection{Parameters}

$D_{i t}$ : Forecast of demand for product $\mathrm{i}$ in period $\mathrm{t}$,

$p_{i t}^{s}$ : The cost of producing one unit of product $\mathrm{i}$ during the period $\mathrm{t}$ in the second

$p_{i t}^{s}: \quad$ stage in normal time,

$o_{i t}^{s}: \quad$ The cost of producing one unit of product $\mathrm{i}$ during the t period in the second stage during overtime,

$s_{i t}^{s}$ : Contractor's cost to supply one unit of product $\mathrm{i}$ in period $\mathrm{t}$ in the second stage,

$b_{i t}^{s}$ : The backorder cost for the product $\mathrm{i}$ in the $\mathrm{t}$ period in the second stage,

$h_{i t}^{s}$ : The holding cost for the product $\mathrm{i}$ in the $\mathrm{t}$ period in the second stage,

$p_{k t}^{f}$ : The production cost in normal time to produce a unit of product $\mathrm{k}$ during the $\mathrm{t}$

period in the first Stage,

$o_{k t}^{f}$ : The production cost of produce one unit of product $\mathrm{k}$ during the $\mathrm{t}$ period in overtime of the first stage,

$s_{k t}^{f}$ : Contractor's cost to supply one unit of product $\mathrm{k}$ in period $\mathrm{t}$ in the first stage,

$h_{k t}^{f}: \quad$ The holding cost for the product $\mathrm{i}$ in the $\mathrm{t}$ period in the first stage,

$a_{i j}^{s}: \quad$ The required time for the $\mathrm{j}$ machine to produce one unit of product $\mathrm{i}$ in the second

$a_{i j} \cdot$ stage,

$a_{k l}^{f}:$ The required time for the 1 machine to produce one unit of product $\mathrm{j}$ in the first stage, 
$u_{i j}^{s}$ : The set-up time for the $\mathrm{j}$ machine to produce one unit of product $\mathrm{i}$ in the second

stage,

$u_{k l}^{f}$ : The set-up time for the 1 machine to produce one unit of product $\mathrm{j}$ in the first

$u_{k l}^{f}$ : stage,

$r_{i j t}^{s}$ : The set-up cost for production of product $\mathrm{i}$ on the machine $\mathrm{j}$ in period $\mathrm{t}$ in second

stage,

$r_{t h}^{f}$ : The set-up cost for production of product $\mathrm{k}$ on the machine 1 in period $\mathrm{t}$ in first stage,

$R_{j t}^{s}$ : Maximum available normal time for machine $\mathrm{j}$ in period $\mathrm{t}$ in second stage,

$R_{l t}^{f}$ : Maximum available normal time for machine 1 in period $\mathrm{t}$ in first stage,

$h r_{t}$ : The hiring cost of a worker in period $\mathrm{t}$,

$l o_{t}$ : The deposal cost of a worker in period $\mathrm{t}$,

$w_{t}$ : The labor cost in the period $\mathrm{t}$,

$I_{i 0}^{s}$ : The initial inventory level for product i (at the beginning of the program horizon)

$I_{i 0} \cdot$ in the t period in the second stage,

$I_{k 0}^{f}$ : The initial inventory level for product $\mathrm{k}$ (at the beginning of the program

horizon) in the t period in the first stage,

$W_{0}$ : The labor level at the start of the planning,

$S_{k 0}^{f}$ : The volume of the contract for product $\mathrm{k}$ at the start of the planning,

$f_{i k}$ : The required units from the product $\mathrm{k}$ in the first stage to produce one unit of

$f_{i k}$ : product i in the second stage,

$e_{i}^{s}$ : The required time by the personnel to produce one unit of product $\mathrm{i}$ in the second

$e_{i}^{s}:$ stage,

$e_{k}^{f}:$ The required time by the personnel to produce one unit of product $\mathrm{k}$ in the first stage,

$\alpha_{t}$ : Ratio of available labor in (normal time) to (overtime) in period t,

$\beta_{j t}^{s}$ : Ratio of available capacity of machine $\mathrm{j}$ in (normal time) to (overtime) in period $\mathrm{t}$ in second stage,

$\beta_{l t}^{f}$ : Ratio of available capacity of machine 1 in (normal time) to (overtime) in period $\mathrm{t}$

in first stage,

$\rho$ : Personnel working hours in each period,

$V_{i}^{s}$ : Occupied capacity to hold each product $\mathrm{i}$ in the second stage,

$V_{k}^{f}$ : Occupied capacity to hold each product i in the first stage,

$I_{\text {Max }}^{s}$ : Total storage capacity of products in the second stage,

$I_{M a x}^{f}$ : Total storage capacity of products in the first stage,

$W_{t}^{\text {Max }}$ : Maximum available labor in period $\mathrm{t}$,

$S_{i t}^{s M a x}$ : The maximum number of available contractors to produce product $\mathrm{i}$ in the $\mathrm{t}$ 
A Robust Optimization Model for Multi-Product Production Planning in Terms of Uncertainty of Demand and Delivery Time

period in the second stage,

$L D_{k t}$ : The delivery lead-time of the product $\mathrm{k}$ from the first stage to the second stage in the period $\mathrm{t}$,

$P T_{k t}^{f}$ : Duration of production of product $\mathrm{k}$ in period $\mathrm{t}$ in the first stage,

$C V_{k t}^{f}$ : The late delivery cost of the product $\mathrm{k}$ from the first stage to the second stage,

$e v_{k t}^{f}:$ Late delivery penalty for delay of one unit of product $\mathrm{k}$ in the first stage 9

$M:$ A positive very large number,

\subsection{Decision variables}

$P_{i t}^{s}$ : The number of product $\mathrm{i}$ that produced during normal time in period $\mathrm{t}$ in the second

$O_{i t}^{s}$ : The number of product $\mathrm{i}$ that produced during over time in period $\mathrm{t}$ in the second

$O_{i t}^{s} \cdot$ stage,

$S_{i t}^{s}$ : The number of contractors for producing product $\mathrm{i}$ in period $\mathrm{t}$ in the second stage,

$B_{i t}^{s}$ : The backorder level of product $\mathrm{i}$ in period $\mathrm{t}$ in the second stage,

$I_{i t}^{s}$ : The inventory level of product $\mathrm{i}$ in period $\mathrm{t}$ in the second stage,

$P_{k t}^{f}$ : The number of product $\mathrm{k}$ that produced during normal time in period $\mathrm{t}$ in the first

$O_{k t}^{f}$ : The number of product k that produced during over time in period $\mathrm{t}$ in the first stage,

$S_{k t}^{f}$ : The number of contractors for producing product k in period $\mathrm{t}$ in the first stage,

$I_{k t}^{f}$ : The inventory level of product $\mathrm{k}$ in period $\mathrm{t}$ in the first stage,

$H_{t}$ : Number of hired labor in period $\mathrm{t}$,

$L_{t}$ : Number of deposal labor in period $\mathrm{t}$,

$W_{t}$ : Labor level in period $\mathrm{t}$,

$Y_{i t}^{s}$ : The decision variable of producing product $\mathrm{i}$ in the $\mathrm{t}$ period in the second stage,

$Y_{k t}^{f}$ : The decision variable of producing product $\mathrm{k}$ in the $\mathrm{t}$ period in the first stage, 
Esmaeil Mehdizadeh, Habibreza Gholami, Bahman Naderi

\subsection{Proposed mathematical model}

The mathematical model is as follows:

$$
\begin{aligned}
& \text { Min } T C=\sum_{t=1}^{T} \sum_{i=1}^{N}\left(p_{i t}^{s} \cdot P_{i t}^{s}+o_{i t}^{s} \cdot O_{i t}^{s}+s_{i t}^{s} \cdot S_{i t}^{s}\right)+\sum_{t=1}^{T} \sum_{i=1}^{N} \sum_{j=1}^{J} r_{i j t}^{s} \cdot Y_{i t}^{s}+\sum_{t=1}^{T} \sum_{i=1}^{N} h_{i t}^{s} \cdot I_{i t}^{s}+\sum_{t=1}^{T} \sum_{i=1}^{N} b_{i t}^{s} \cdot B_{i t}^{s}+ \\
& \sum_{t=1}^{T} \sum_{k=1}^{K}\left(p_{k t}^{f} \cdot P_{k t}^{f}+O_{k t}^{f} \cdot O_{k t}^{f}+s_{k t}^{f} \cdot S_{k t}^{f}\right)+\sum_{t=1}^{T} \sum_{k=1}^{K} \sum_{l=1}^{L} r_{k l t}^{f} \cdot Y_{k t}^{f}+\sum_{t=1}^{T} \sum_{k=1}^{K} h_{k t}^{f} \cdot I_{k t}^{f}+ \\
& \sum_{t=1}^{T}\left(h r_{t} \cdot H_{t}+l o_{t} \cdot L_{t}++w_{t} \cdot W_{t}\right)+\sum_{t=1}^{T} \sum_{k=1}^{K} \alpha_{k t}^{f} \cdot Y_{k t}^{f} \\
& S . T: \\
& \quad P_{i t}^{s}+O_{i t}^{s}+S_{i t}^{s}+B_{i t}^{s}-B_{i(t-1)}^{s}+I_{i(t-1)}^{s}-I_{i t}^{s}=D_{i t} \\
& P_{k t}^{f}+O_{k t}^{f}+S_{k t}^{f}+I_{k(t-1)}^{f}-I_{k t}^{f}=\sum_{i=1}^{N} f_{i k}\left(P_{i t}^{s}+O_{i t}^{s}\right) \\
& S_{k 0}^{f}+I_{k 0}^{f}=\sum_{t=1}^{L-1} \sum_{i=1}^{N} f_{i k}\left(P_{i t}^{s}+O_{i t}^{s}\right) \\
& \sum_{i=1}^{N}\left(\alpha_{i j}^{s} \cdot P_{i t}^{s}+u_{i j}^{s} \cdot Y_{i t}^{s}\right) \leq R_{j t}^{s} \\
& \sum_{i=1}^{N} \alpha_{i j}^{s} \cdot O_{i t}^{s} \leq \beta_{j t}^{s} \cdot R_{j t}^{s} \\
& \sum_{k=1}^{K}\left(\alpha_{k l}^{f} \cdot P_{k t}^{f}+u_{k l}^{f} \cdot Y_{k t}^{f}\right) \leq R_{l t}^{f} \\
& \sum_{k=1}^{K} \alpha_{k l}^{f} \cdot O_{k t}^{f} \leq \beta_{l t}^{f} \cdot R_{l t}^{f} \\
& P_{i t}^{s}+O_{i t}^{s} \leq M \cdot Y_{i t}^{s} \\
& P_{k t}^{f}+O_{k t}^{f} \leq M \cdot Y_{k t}^{f} \\
& W_{t}=W_{t-1}+H_{t}-L_{t} \\
& \sum_{i=1}^{N} \alpha_{i}^{s} \cdot P_{i t}^{s}+\sum_{k=1}^{K} e_{k}^{f} \cdot P_{k t}^{f} \leq \rho \cdot W_{t} \\
& \sum_{i=1}^{N} e_{i}^{s} \cdot O_{i t}^{s}+\sum_{k=1}^{K} e_{k}^{f} \cdot O_{k t}^{f} \leq \alpha_{t} \cdot \rho \cdot W_{t} \\
&
\end{aligned}
$$


A Robust Optimization Model for Multi-Product Production Planning in Terms of Uncertainty of Demand and Delivery Time

$\sum_{i=1}^{N} V_{i}^{s} \cdot I_{i t}^{s} \leq I_{M a x}^{s}$

$\sum_{k=1}^{k} V_{k}^{f} \cdot I_{k t}^{f} \leq I_{M a x}^{f}$

$W_{t} \leq W_{t}^{\operatorname{Max}}$

$S_{l t}^{s} \leq S_{i t}^{s \operatorname{Max}}$

$B_{i t}^{s} \cdot I_{i t}^{s}=0$

$H_{t} \cdot L_{t}=0$

$C V_{k t}^{f}=\operatorname{Max}\left(\left(\left(I D_{k t}^{f}+P T_{k t}^{f}\right)-I D \max _{k t}\right) e v_{k t}, 0\right) \quad \forall k, t$

$P_{i t}^{s}, O_{i t}^{s}, S_{i t}^{s}, B_{i t}^{s}, I_{i t}^{s}, P_{k t}^{f}, O_{k t}^{f}, S_{k t}^{f}, I_{k t}^{f}, H_{t}, L_{t}, W_{t}, P T_{k}^{f}, I D_{k t} \geq 0 \quad ; \quad B_{i t}^{s}=0$

$Y_{i t}^{s} . Y_{k t}^{f}=\{0,1\}$

In the objective function (equation 1), the first part calculates the total production cost, taking into account the normal time and the overtime of production and contract costs for the products of the second stage. Parts two to four are set up, inventory, and delay the delivery cost for the second stage of the production. The fifth part is the total cost of production for the products in the first stage, the sixth is the total set-up cost in the first stage of the product and the seventh part is the total maintenance cost in the first stage, taking into account the number of products stored in the warehouse at a time interval. The eighth part is the total cost of employing, hiring and deposal the workforce, taking into account the different levels of work, and the final part is the late delivery of the product of the first stage to the second stage of production.

Equation (2) is about satisfaction of demand for products in the second production stage. The Equation (3) guaranteed the balance of production and inventory and lateral products of the first and second stage of production. Equation (4) represents that the initial inventory level of the products of the first stage at the start of the process should be greater than the total of the products manufactured in the first $(l)$ period in the second stage to satisfy the demand for the product. Equations (5) and (6) determine that the normal working time and overtime of production are limited to the availability of machines of the second stage production, according 
Esmaeil Mehdizadeh, Habibreza Gholami, Bahman Naderi

into their capacity. Through equations (7) and (8), the total production in the first stage is limited according into the limitations of production capacity at normal times and overtime in the first stage of production. Equations (9) and (10) establish the relationship between the production variables and the launching of the first and second stages of production. Equation (11) is the level of work classification for both groups of workers. Equations (12) and (13) are related to the limitation of access to labor in normal and overtime. Equations (14) and (15) are the restrictions on occupation of space by the products in first and second production stages. Equation (16) limits the classification of jobs to existing labors. Equation (17) limits the levels of contractors and lateral products to current levels. Naturally, equations 18 and 19 are not necessary to minimize the target function, and we can ignore them. Equation (20) means that in the event of late delivery of product from stage one to stage two and failure to comply with delivery time, a penalty will be paid. Finally, equations (21) and (22) define the parameters and the problem variables.

\section{Introducing the robust optimization model}

A robust optimization is used to provide a set of solutions that stays stable against some future parameters fluctuation (input data). A robust optimization approach was presented by Mulvey et al. [7]. The general framework for such optimization is briefly described below. The basic linear programming model is as follows:

$$
\begin{aligned}
& \operatorname{Min} c^{T} \cdot x+d^{T} \cdot y \\
& \text { S.T: } \\
& \quad \text { A. } x=b \\
& \quad \text { B. } x+C \cdot y=e \\
& \quad x, y \geq 0
\end{aligned}
$$

Where $\mathrm{X}$ is the vector of decision variables and $\mathrm{Y}$ is the vector of control variables. The equation (24) is a basic limitation in which the coefficients are definite. Equation (25) is a control constraint, some of which are non-deterministic. In robust optimization, non-deterministic parameters are modeled by a set of scenarios. As a result, the set $\left\{B^{\xi}, C^{\xi}, e^{\xi}, d^{\xi}\right\}$ is a set of non-deterministic parameters under each of the scenarios, and $\sum p^{\xi}=1$ is where $p^{\xi}$ is the 
A Robust Optimization Model for Multi-Product Production Planning in Terms of Uncertainty of Demand and Delivery Time

probability of $\varepsilon$ scenario. The robust scenario-based optimization approach is as follows:

$$
\operatorname{Min} c^{T} \cdot x+\sum_{\xi \in \Omega} d^{T \xi} \cdot y^{\xi}+\lambda \cdot \sigma\left(y^{1}, y^{2}, \ldots, y^{\Xi}\right)+\omega . \rho\left(\delta^{1}, \delta^{2}, \ldots, \delta^{\Xi}\right)
$$

S.T:

$$
\begin{aligned}
& A \cdot x=b \\
& B^{\xi} \cdot x+C^{\xi} \cdot y+\delta^{\xi}=e^{\xi} \\
& x, y^{\xi} \geq 0
\end{aligned}
$$

The purpose of this model is to balance the stability of the solving and stability of the model. The optimal solution of this model is optimal when for each scenario the optimal value is near to the initial optimality. It can also be solved if, for any possible scenario, the space of the model is almost possible. For model stability, parameter $\delta^{\xi}$ is defined, which determines the allowable impossibility of control constraints under scenario $\varepsilon$. For more information, we can look at the models presented by Mulvey et al [7].

\section{The proposed robust optimization model}

In the study of the two-stage production system of this paper, the demand and delivery time of the product are uncertain and scenario-based. The model is as follows:

$\operatorname{Min} \sum_{\xi=1}^{\Xi} p^{\xi} . T C^{\xi}+\lambda \sum_{\xi=1}^{\Xi} p^{\xi}\left|T C^{\xi}-\sum_{\xi=1}^{\Xi} p^{\xi} . T C^{\xi}\right|+\omega \sum_{\xi=1}^{\Xi} \sum_{t=1}^{T} \sum_{n=1}^{N} p^{\xi} \cdot \delta_{i t \xi}$

$S . T:$ 


$$
\begin{aligned}
& T C^{\xi}=\sum_{t=1}^{T} \sum_{i=1}^{N}\left(p_{i t}^{s} \cdot P_{i t}^{s}+o_{i t}^{s} \cdot O_{i t}^{s}+s_{i t}^{s} \cdot S_{i t}^{s}\right)+\sum_{t=1}^{T} \sum_{i=1}^{N} \sum_{j=1}^{J} r_{i j t}^{s} \cdot Y_{i t}^{s}+\sum_{t=1}^{T} \sum_{i=1}^{N} h_{i t}^{s} \cdot I_{i t}^{s}+\sum_{t=1}^{T} \sum_{i=1}^{N} b_{i t}^{s} \cdot B_{i t}^{s}+ \\
& \sum_{t=1}^{T} \sum_{k=1}^{K}\left(p_{k t}^{f} \cdot P_{k t}^{f}+o_{k t}^{f} \cdot O_{k t}^{f}+s_{k t}^{f} \cdot S_{k t}^{f}\right)+\sum_{t=1}^{T} \sum_{k=1}^{K} \sum_{l=1}^{L} r_{k l t}^{f} \cdot Y_{k t}^{f}+\sum_{t=1}^{T} \sum_{k=1}^{K} h_{k t}^{f} \cdot I_{k t}^{f}+ \\
& \sum_{t=1}^{T}\left(h r_{t} \cdot H_{t}+l o_{t} \cdot L_{t}++w_{t} \cdot W_{t}\right)+\sum_{t=1}^{T} \sum_{k=1}^{K} \alpha_{k t}^{f} \cdot Y_{k t}^{f} \\
& P_{i t}^{s}+O_{i t}^{s}+S_{i t}^{s}+B_{i t \xi}^{s}-B_{i(t-1) \xi}^{s}+I_{i(t-1) \xi}^{s}-I_{i t \xi}^{s}=D_{i t \xi}
\end{aligned}
$$

Equations(3) to (22)

\section{Solving the proposed robust model}

To solve the presented model, at first seven problems generated randomly when condition is not robust and the presented model solved by B\&B method by using Lingo software in $1800 \mathrm{Sec}$. The results are shown in Table 1. Finally for solving the proposed robust model, data extracted from a Pasta company was used. In this regard, using the knowledge of the experts and the access to historical data of the company as well as economic conditions, three scenarios are described as optimistic, probabilistic and pessimistic. According to the collected data, the probabilities of occurrence of each scenario are $0.35,0.4$ and 0.25 , respectively. The planning horizon is also 6 months. Information about scenarios is shown in

Tables 2 and 3. 
A Robust Optimization Model for Multi-Product Production Planning in Terms of Uncertainty of Demand and Delivery Time

Table 1. Solving time of problems

\begin{tabular}{ccccccccc}
\hline & & & & & \multicolumn{3}{c}{ LINGO } \\
\cline { 6 - 8 } & & & & & & LB & Obj.func. & time (s) \\
\hline 1 & 1 & 2 & 2 & 1 & 3 & 115278 & 115646 & 1800 \\
2 & 1 & 3 & 2 & 1 & 3 & 262201 & 262201 & 1800 \\
3 & 1 & 4 & 2 & 1 & 3 & 609772 & 610401 & 1800 \\
4 & 1 & 2 & 2 & 1 & 6 & 12910500 & 13302600 & 1800 \\
5 & 1 & 3 & 2 & 1 & 6 & 15626400 & 15638400 & 1800 \\
6 & 1 & 3 & 2 & 2 & 3 & 3724630 & 3728280 & 1800 \\
7 & 1 & 3 & 2 & 2 & 6 & 9774750 & 12188100 & 1800 \\
\hline
\end{tabular}

Table 2. Demand for different scenarios

\begin{tabular}{cccccccc} 
& & \multicolumn{5}{c}{ Demand (Ton) } \\
\cline { 3 - 7 } Production & \multirow{2}{*}{ Scenario } & $1^{\text {st }}$ & $2^{\text {nd }}$ & $3^{\text {rd }}$ & $4^{\text {st }}$ & $5^{\text {st }}$ & $6^{\text {st }}$ \\
\cline { 3 - 7 } & Optimistic & 1200 & 2350 & 2700 & 2700 & 1700 & 1700 \\
\multirow{2}{*}{1} & Probabilistic & 1624 & 2657 & 3334 & 3133 & 2086 & 2007 \\
& Pessimistic & 2335 & 3096 & 3545 & 3479 & 3297 & 3251 \\
& Optimistic & 2700 & 10200 & 10200 & 9200 & 9200 & 8700 \\
2 & Probabilistic & 3435 & 11534 & 13133 & 11732 & 9549 & 9578 \\
& Pessimistic & 3696 & 12586 & 13177 & 13948 & 13912 & 13912 \\
& Optimistic & 100 & 100 & 150 & 150 & 200 & 200 \\
\multirow{2}{*}{3} & Probabilistic & 150 & 300 & 300 & 400 & 400 & 450 \\
& Pessimistic & 300 & 500 & 500 & 650 & 650 & 650 \\
\hline
\end{tabular}


Esmaeil Mehdizadeh, Habibreza Gholami, Bahman Naderi

Table 3.Lead time for different scenarios

\begin{tabular}{cccccccc} 
& & \multicolumn{5}{c}{ Lead time (Months) } \\
\hline \multirow{2}{*}{ Production } & Scenario & $1^{\text {st }}$ & $2^{\text {nd }}$ & $3^{\text {rd }}$ & $4^{\text {st }}$ & $5^{\text {st }}$ & $6^{\text {st }}$ \\
\hline \multirow{2}{*}{1} & Optimistic & 10 & 20 & 23 & 23 & 15 & 15 \\
& Probabilistic & 14 & 23 & 29 & 27 & 18 & 17 \\
& Pessimistic & 20 & 27 & 31 & 30 & 29 & 28 \\
2 & Optimistic & 5 & 20 & 20 & 18 & 18 & 17 \\
2 & Probabilistic & 7 & 23 & 26 & 23 & 19 & 19 \\
& Pessimistic & 7 & 25 & 26 & 28 & 28 & 28 \\
& Optimistic & 3 & 3 & 5 & 5 & 7 & 7 \\
3 & Probabilistic & 5 & 10 & 10 & 13 & 13 & 15 \\
& Pessimistic & 10 & 17 & 17 & 22 & 22 & 22 \\
\hline
\end{tabular}

\subsection{Comparison of robust model with average of 3 scenarios}

In this section, the average of the three scenarios was calculated and then the comparative graph with the robust model was presented. As shown in figure 1, the objective function for the robust model is less oscillating than the normal one.

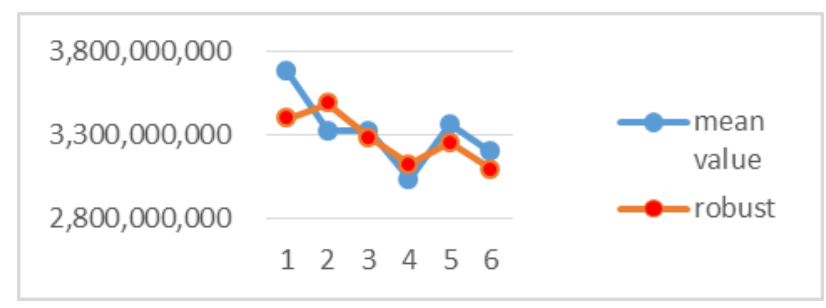

Figure 1. Comparison between robust and mean value

\subsection{Exchanges between solution robustness and model robustness}

The parameter $\varpi$ in the robust model is referred to as the parameter for setting the exchange between risk and cost. This means that according to the decisionmaker's circumstances and opinions, the degree of robustness of the solution (the closeness of the ideal solution to the conditions of the existing uncertainty) and the degree of robustness of the model (the validity of the solution) will be tunable [4]. If $\varpi=0$, then the unmet demand will equal the total market demand, in which case the production rate of each product will be zero and the objective function will be the lowest possible value. Hence, in order to achieve a feasible and optimal solution, the robust model should be solved for different values of $\varpi$ and the interactions between the model's robustness and the solution must analyze. These 
A Robust Optimization Model for Multi-Product Production Planning in Terms of Uncertainty of Demand and Delivery Time

results are presented in table 4 . As shown in this table, with the increase in the amount of $\varpi$, the total cost that represents the degree of robustness of the solution has increased, while the unmet demand is decreasing with the increase of $\varpi$. Also, for large $\varpi$ values, the solutions given for each of the possible scenarios is often feasible, but it will lead to more costs. Meanwhile, the results are consistent with the results presented by Mulvey et al. [7]. Regarding these items, the choice of the $\varpi$ parameter in the robust model depends on the decision maker's view of the exchange between risk and cost. In this study, the amount of $\varpi$ was considered to be 400 . In these circumstances, almost $20 \%$ of the demand is not met.

Table 4.Compare the answer for different values of $\varpi$.

\begin{tabular}{ccc}
\hline $\begin{array}{c}\text { Increased } \\
\text { percentage of } \\
\text { objective function }\end{array}$ & $\begin{array}{c}\text { Solution } \\
\text { feasibility } \\
\text { probability }\end{array}$ & $\varpi$ \\
\hline- & 0 & 0 \\
0.07 & $15 \%$ & 50 \\
0.53 & $21 \%$ & 100 \\
0.89 & $29 \%$ & 150 \\
1.26 & $38 \%$ & 200 \\
1.67 & $53 \%$ & 250 \\
2.11 & $62 \%$ & 300 \\
2.53 & $71 \%$ & 350 \\
2.94 & $79 \%$ & 400 \\
\hline
\end{tabular}

\section{Conclusion}

In this paper, a multi-product multi-period planning model was presented in the uncertainty conditions of some of the parameters. The objective function of presented model was minimizing the cost of production in the planning horizon and this model was solved by using the Branch and Bound (B\&B) method. In this model, in order to confronting with the uncertainty of the data and reduce their effects on the objective function, a robust optimization model was used that proposed by Mulvey et al. [7]. In their model, parameters with uncertainty are defined as a scenario. In this paper, three optimistic, pessimistic and probabilistic scenarios were considered for uncertain data. Then the objective function was obtained for different scenarios and compared with the result of the robust optimization model. As can be seen from the results, the results of the robust model, for the different periods, have a lower oscillation than the non-robust 
Esmaeil Mehdizadeh, Habibreza Gholami, Bahman Naderi

model. This shows that the proposed model is useful for systems, which in addition to the cost of production, the robustness of the solution is also important for its managers. In fact, for such systems, it's not just a solution to the problem with the least cost, but it is also important to reduce the fluctuations resulting from the uncertain data on the optimal solution. For the development of this model and as the further studies, one can consider other uncertain parameters in the model. It can also be solved using other robust techniques. In this research, a B\&B method was used to solve the presented model, although meta-heuristic algorithms such as evolutionary algorithms can be used to compare the results with this paper.

\section{REFERENCES}

[1] Wang, R. and H. Fang (2000), Aggregate Production Planning in a Fuzzy Environment. International Journal of Industrial EngineeringTheory, Applications and Practice, 7(1): pp. 5-14;

[2] Leung, S., Y. Wu and K. Lai (2006), A Stochastic Programming Approach for Multi-site Aggregate Production Planning. Journal of the Operational Research Society, 57(2): pp. 123-132;

[3] Ramezanian, R., D. Rahmani and F. Barzinpour(2012), An Aggregate Production Planning Model for Two Phase Production Systems: Solving with Genetic Algorithm and Tabu Search. Expert Systems with Applications, 39(1): pp. 1256-1263;

[4] Leung, S.C. and Y. Wu (2004), A Robust Optimization Model for Stochastic Aggregate Production Planning. Production planning \& control, 15(5): pp. 502-514;

[5] Kastsian, D. and M. Mönnigmann(2014), Robust Optimization of Periodically Operated Nonlinear Uncertain Processes. Chemical Engineering Science, 106: pp. 109-118;

[6] Sakhaii, M., et al. (2016), A Robust Optimization Approach for an Integrated Dynamic Cellular Manufacturing System and Production Planning with Unreliable Machines. Applied Mathematical Modelling, 40(1): pp. 169-191;

[7] Mulvey, J.M., R.J. Vanderbei and S.A. Zenios(1995), Robust Optimization of Large-scale Systems. Operations research, 43(2): pp. 264-281. 